\title{
Short communication: Effect of commercial or depurinized milk diet on plasma advanced oxidation protein products, cardiovascular markers, and bone marrow $\mathrm{CD}_{34}{ }^{+}$stem cell potential in rat experimental hyperuricemia
}

\author{
Gordana Kocic, ${ }^{* 1}$ Dusan Sokolovic, ${ }^{*}$ Tatjana Jevtovic, ${ }^{*}$ Tatjana Cvetkovic, ${ }^{*}$ Andrej Veljkovic, ${ }^{*}$ \\ Hristina Kocic, $†$ Svetlana Stojanovic, ${ }^{*}$ Aneta Jovanovic, $\ddagger$ Jelena Jovanovic, $\ddagger$ and Petar Zivkovic \\ *Medical Faculty University Nis, Institute of Biochemistry, Bul Dr Zorana Djindjica 81, Nis 18000, Serbia \\ †Medical Faculty, University Maribor, Maribor 2000, Magdalenski trg 5, Slovenia \\ ¥Medical Faculty, University Nis, Bul Dr Zorana Djindjica 81, Nis 18000, Serbia
}

\begin{abstract}
Cardiovascular repair and myocardial contractility may be improved by migration of bone marrow stem cells (BMSC) and their delivery to the site of injury, a process known as BMSC homing. The aim of our study was to examine the dietary effect of a newly patented depurinized milk (DP) that is almost free of uric acid and purine and pyrimidine compounds compared with a standard commercial $1.5 \%$ fat UHT milk diet or allopurinol therapy in rat experimental hyperuricemia. Bone marrow stem cell potential (BMCD34 ${ }^{+}$, CD34postive bone marrow cells), plasma oxidative stress parameters [advanced oxidation protein products, AOPP) and thiobarbituric acid reactive substances (TBARS)], myocardial damage markers [creatine phosphokinase $(\mathrm{CPK})$, aspartate aminotransferase (AST), and lactate dehydrogenase $(\mathrm{LDH})$ ], plasma cholesterol, and highdensity lipoprotein cholesterol were investigated. The DP milk diet significantly increased the number of BMCD $34^{+}$stem cells compared with commercial UHT milk. Allopurinol given alone also increased the number of BMCD34 ${ }^{+}$. Hyperuricemia caused a significant increase in all plasma enzyme markers for myocardial damage (CPK, LDH, and AST). A cardioprotective effect was achieved with allopurinol but almost equally with DP milk and more than with commercial milk. Regarding plasma AOPP, TBARS, and cholesterol levels, the most effective treatment was DP milk. In conclusion, the protective role of a milk diet on cardiovascular function may be enhanced through the new depurinized milk diet, which may improve cardiovascular system function via increased bone marrow stem cell regenerative potential, decreased plasma oxidative stress parameters, and decreased levels of myocardial damage markers and cholesterol. New dairy technol-
\end{abstract}

Received July 1, 2014

Accepted August 8, 2014.

${ }^{1}$ Corresponding author: kocicrg@yahoo.co.uk ogy strategies focused on eliminating harmful milk compounds should be completely nontoxic. Novel milk products should be tested for their ability to improve tissue repair and function.

Key words: milk, hyperuricemia, advanced oxidation protein products (AOPP), bone marrow $\mathrm{CD} 34^{+}$, cardiovascular marker

\section{Short Communication}

Bone marrow stem cells (BMSC) possess the ability to differentiate into cardiac myocytes, endothelial cells, or vascular smooth muscle cells. Myocardial regeneration and contractility may be improved by administered or mobilized BMSC, which is known as BMSC repopulation therapy (Jackson et al., 2001; Orlic et al., 2001, 2002).

Beside primary hyperuricemia, which is usually present in gout, hyperuricemia has also been documented in cardiovascular diseases and hypertension. Hyperuricemia can aggravate prognosis and mortality of myocardial infarction, ischemic cardiomyopathy, and congestive heart failure (Fang and Alderman, 2000; Feig et al., 2008; Ioachimescu et al., 2008; Strasak et al., 2008). Whether hyperuricemia has a causal role in these syndromes or represents a secondary phenomenon is unknown; urate crystals have been found within atherosclerotic plaques together with cholesterol. Hyperuricemia may contribute to atherosclerotic plaque progression (Dunkelgrun et al., 2008; Tavil et al., 2008). Dairy products are recommended in management of hyperuricemia (Dalbeth et al., 2010). We patented a new milk formula almost free of purine and pyrimidine compounds and uric acid - depurinized (DP) milk (Kocic et al., 2010, 2011; Carluccio and Kocic, 2011; Cencic et al., 2011]. Our previous results documented the beneficial effects of a DP milk diet regimen over 3 and $10 \mathrm{~d}$, when given together with a standard laboratory chow or alone, on plasma uric acid, lipid parameters, liver regenerative potential, and antiapoptotic effects in rats, 
as investigated through the key signaling molecules and endonuclease activity (Kocic et al., 2012, 2014).

Our current study examined the effect of milk dietary regimen (standard commercial $1.5 \%$ fat UHT milk or DP milk) compared with standard laboratory chow and allopurinol therapy in experimental hyperuricemia, regarding BMSC potential, oxidative stress parameters [advanced oxidation protein products (AOPP) and lipid peroxidation products (thiobarbituric acid reactive substances; TBARS)], myocardial damage markers (creatine phosphokinase (CPK), aspartate aminotransferase (AST), and lactate dehydrogenase (LDH)], cholesterol and high-density lipoprotein (HDL) cholesterol level.

Oxonic acid was purchased from Sigma Aldrich (St. Louis, MO) and allopurinol was purchased from Zorka Pharma (Šabac, Serbia). Depurinized milk production and experimental design was explained in a previous article (Kocic et al., 2014). Briefly, experimental hyperuricemia in rats was induced by administering potassium oxonate dissolved in drinking water in a daily dose of $100 \mathrm{mg}$. Each experimental group (6 rats per group) was on the assigned dietary regimen for $10 \mathrm{~d}$, and the control group (6 rats) was fed only standard laboratory chow. The allocated groups of rats were (1) control group; (2) rats with induced experimental hyperuricemia; (3) rats with induced experimental hyperuricemia treated with allopurinol (daily dose of $20 \mathrm{mg}$ ); (4) rats with induced experimental hyperuricemia fed DP milk instead of standard laboratory chow; (5) rats with induced experimental hyperuricemia fed control milk instead of standard laboratory chow; (6) untreated healthy rats fed DP milk only instead of standard laboratory chow; (7) rats fed commercial (control) milk only; (8) rats fed allopurinol given in dose of $20 \mathrm{mg}$ and standard laboratory chow. The animals were killed under ketamine anesthesia after $10 \mathrm{~d}$. Blood was collected from the abdominal aorta of each animal in heparinized $15-\mathrm{mL}$ tubes. Plasma was obtained by removing cells by centrifugation at $2,000 \times g$ for 10 minutes at $4^{\circ} \mathrm{C}$ using a refrigerated centrifuge (Allegra 64R, Beckman Coulter, Pasadena, CA).

For each animal, the head of a single femur was cut off and marrow was removed by aspirating PBS through a puncture in the other end of the bone using a needle and syringe. The marrow recovered from one femur was suspended in $1 \mathrm{~mL}$ of PBS, placed in an 80 -mesh stainless steel sieve, and kept at $-20^{\circ} \mathrm{C}$ before analysis.

Uric acid (UA), AOPP, lipid peroxidation (TBARS), total cholesterol, HDL cholesterol, and myocardial enzymes (CPK, AST, and LDH) were analyzed in plasma by using a Dade Behring RxL Max automatic analyzer (Dade Behring, Deerfield, IL) and Beckman DU640 spectrophotometer (Beckman Coulter). The AOPP were measured in the supernatant at $340 \mathrm{~nm}$ under acidic conditions and expressed as chloramine- $\mathrm{T}$ equivalents (Marsche et al., 2009). Malondialdehyde (MDA) was determined in samples via reaction with thiobarbituric acid, which generates MDA-thiobarbituric acid adducts (TBARS), which were quantified spectrophotometrically at $532 \mathrm{~nm}$ (Yagi, 1998).

For determination of CD34 ${ }^{+}$antigen, $10 \mu \mathrm{L}$ of each animal's bone marrow sample was pipetted into 2 flatbottomed, 96-well polystyrene plates (test and control plate), according to a method reported previously (Kocic et al., 2014). A CD34 mouse monoclonal $\mathrm{IgG}_{1}$ antibody (ICO115) was purchased from Santa Cruz Biotechnology (Santa Cruz, CA). Mean fluorescence intensity (logarithmic scale) was determined by using a Victor Perkin Elmer-Wallac multiplate reader (PerkinElmer, Waltham, MA).

All data were expressed as mean \pm standard deviation (SD). The comparison between the dietary groups for each parameter analyzed was performed using ANOVA. Differences were considered statistically significant at $P<0.05$.

Results are shown in Table 1. Oxonic acid tended to decrease BMCD34 ${ }^{+}$level but this effect was not significant. The DP milk diet significantly increased BMCD $34^{+}$stem cell potential compared with commercial milk. The most effective treatment was when allopurinol was given to healthy animals fed standard laboratory chow (group 8). The AOPP level was significantly reduced in all investigated groups, but especially in the group with experimental hyperuricemia fed DP milk diet (group 4) compared with the control group of healthy rats fed standard laboratory chow (group 1). Experimental hyperuricemia induced by oxonic acid resulted in a significant increase of all plasma myocardial markers (CPK, LDH, and AST). The DP milk diet given to hyperuricemic rats (group 4) resulted in a significant decrease in CPK and LDH compared with the hyperuricemic group (group 2). The DP milk diet given to healthy animals (group 6) resulted in a significant decrease in CPK and LDH compared with that in control healthy animals fed standard laboratory chow (group 1). Because the elevations in enzymes CPK, AST, and LDH can be result of myocardial cellular damage, the results obtained may suggest potential cardioprotective effect of DP milk diet. The obtained effect was significant for the DP milk. Feeding DP milk decreased the activity of all mentioned enzymes in intact animals compared with control values, which was not achieved when giving commercial milk or allopurinol to intact animals. Significantly decreased levels of total cholesterol and HDL cholesterol were observed in all investigated groups compared with control. 


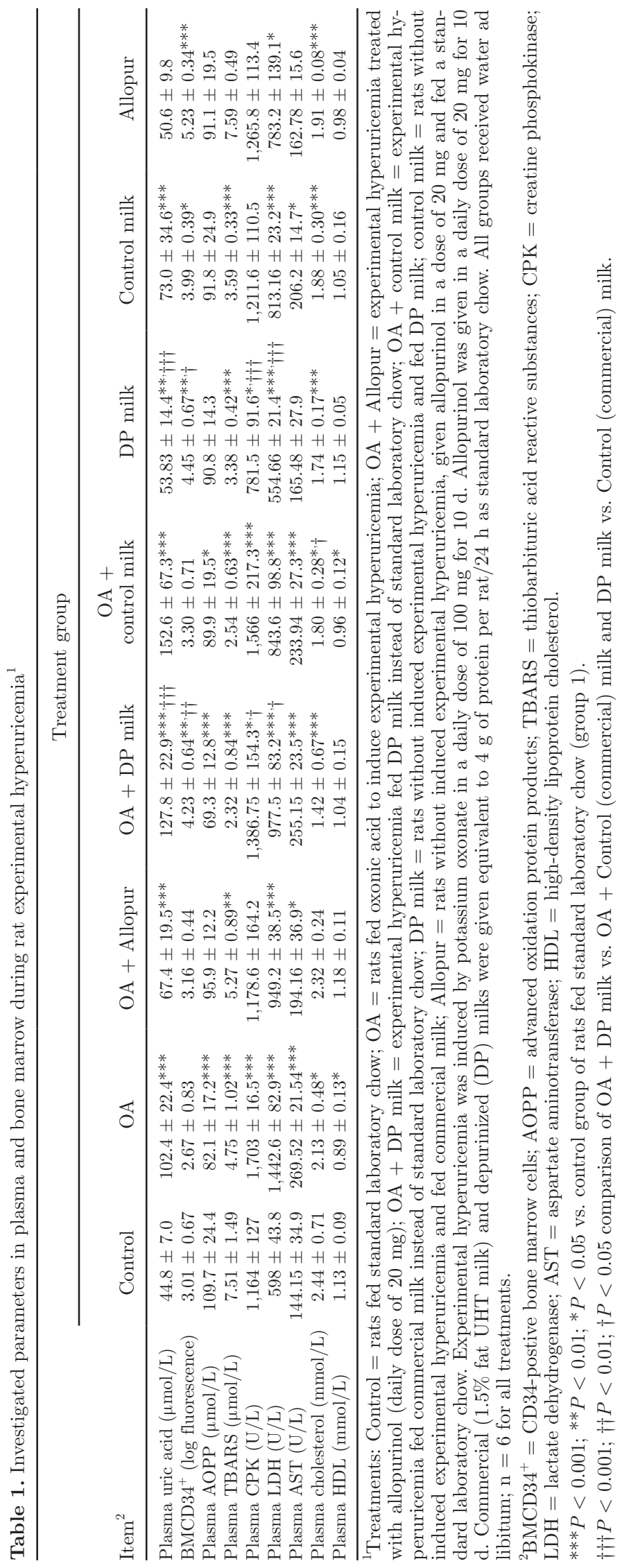


In the current study, we documented that the DP milk dietary regimen, compared with standard commercial UHT cow milk or allopurinol therapy, exerted a significant cardioprotective effect in rats with experimental hyperuricemia induced by oxonic acid, as shown by bone marrow stem cell potential, myocardial and oxidative stress parameters, AOPP and TBARS, plasma cholesterol, HDL cholesterol, and myocardial damage markers (Table 1).

There is a lack of experimental evidence for the mechanism by which uric acid may cause cardiovascular disease and myocardial damage (Fang and Alderman, 2000; Feig et al., 2008; Ioachimescu et al., 2008; Strasak et al., 2008). Uric acid may induce inflammation by activation of antigen-presenting cells, by synthesis of acute plasma proteins (C-reactive protein), monocyte chemoattractant protein-1, or IL-1 $\beta$, or by increased activity of $\mathrm{CD}^{+} \mathrm{T}$ cells (Foell et al., 2007; Cheng et al., 2010). The pathogenesis of hypertension and renal damage may involve a urate crystal-independent mechanism, by activation of angiotensin II and downregulation of nitric oxide synthase (NOS1; Enomoto et al., 2002; Hayden and Tyagi, 2004; Corry et al., 2008). Plasma proteins and lipoproteins are very susceptible to oxidation by reactive oxygen species, but hyperuricemic conditions decreased AOPP and TBARS levels, most probably because of the antioxidative effects of uric acid (Table 1). Our results demonstrate that hyperuricemia caused myocardial damage, with subsequent increase of serum myocardial markers, whereas the DP milk and commercial milk diets decreased plasma AOPP and TBARS levels, emphasizing the protective role of a milk diet in reducing production of reactive oxygen species and their damaging consequences. Our primary finding was that experimental hyperuricemia resulted in elevation of myocardial enzymes and decreased BMCD34 ${ }^{+}$stem cell potential. Taken together, these results indicate that hyperuricemia might potentiate myocardial damage by decreasing recruitment of hematopoietic stem cells from bone marrow, cells that are needed for cardiomyocyte regeneration. Endothelial progenitor cells (EPC) represent a population of circulating stem cells mobilized from bone marrow, with the ability to differentiate into endothelial cells. Uric acid is able to induce mobilization of EPC from the bone marrow in the first hours of acute hyperuricemia, and the EPC remain detectable in blood until 1 wk of persisting hyperuricemia, but severe hyperuricemia for longer periods suppresses mobilization of EPC from the bone marrow (Patschan et al., 2007). Beneficial effects of DP milk compared with commercial milk or a standard (vegetarian) chow were achieved for all investigated variables, especially for myocardial enzymes and BMCD34 ${ }^{+}$stem cell potential (Table 1). In our study, allopurinol therapy also reduced myocardial damage and increased BMCD34 ${ }^{+}$stem cell potential.

In conclusion, considering the damaging effect of persisting hyperuricemia, the protective role of a milk diet, especially in a depurinized form, may improve myocardial and cardiovascular system function via increased potential for BMSC regeneration and increased plasma antioxidation, as well as a decrease in levels of myocardial damage markers.

\section{ACKNOWLEDGMENTS}

The experimental and laboratory work was supported by the Ministry of Science and Technology of the Republic of Serbia (TR 31060).

\section{REFERENCES}

Orlic, D., J. Kajstura, S. Chimenti, I. Jakoniuk, S. M. Anderson, B. Li, J. Pickel, R. McKay, B. Nadal-Ginard, D. M. Bodine, A. Leri, and P. Anversa. 2001. Bone marrow cells regenerate infarcted myocardium. Nature 410:701-705. http://dx.doi.org/10.1038/35070587.

Carluccio, F., and G. Kocic. 2011. Procedimento technologico per de la producone di un alimentare dietetico composito a base di latte con livello ridoto di acido urico e purine e prodoto alimentare cosi ottenuto. F. Carluccio and G. Kocic, assignees. Italy patent no. IT2009PR00057 20090723.

Cencic, A., G. Kocic, G. Nikolic, D. Stojanovic, R. Pavlovic, T. Jevtovic-Stoimenov, D. Sokolovic, S. Stojanovic, S. Pajovic, R. Kocic, F. Carluccio, I. Krajnc, and A. Murua. 2011. Composite dietetic milk draught and method for production of composite milk draught with reduced level of uric acid and purine compounds. A. Cencic, assignee. Slovenia patent no. WO2011037545 (A1) 201103-31.

Cheng, T. H., J. W. Lin, H. H. Chao, Y. L. Chen, C. H. Chen, P. Chan, and J. C. Liu. 2010. Uric acid activates extracellular signalregulated kinases and thereafter endothelin-1 expression in rat cardiac fibroblasts. Int. J. Cardiol. 139:42-49.

Corry, D. B., P. Eslami, K. Yamamoto, M. D. Nyby, H. Makino, and M. L. Tuck. 2008. Uric acid stimulates vascular smooth muscle cell proliferation and oxidative stress via the vascular renin-angiotensin system. J. Hypertens. 26:269-275.

Dalbeth, N. S., D. Greg, G. D. A. Gamble, B. Mason, B. Pool, L. Fairbanks, F. M. McQueen, J. Cornish, I. R. Reid, and K. Palmano. 2010. Acute effect of milk on serum urate concentrations: A randomised controlled crossover trial. Ann. Rheum. Dis. 69:16771682.

Dunkelgrun, M., G. M. Welten, D. Goei, T. A. Winkel, O. Schouten, R. T. van Domburg, Y. R. van Gestel, W. J. Flu, S. E. Hoeks, J. J. Bax, and D. Poldermans. 2008. Association between serum uric acid and perioperative and late cardiovascular outcome in patients with suspected or definite coronary artery disease undergoing elective vascular surgery. Am. J. Cardiol. 102:797-801.

Enomoto, A., H. Kimura, A. Chairoungdua, Y. Shigeta, P. Jutabha, S. H. Cha, M. Hosoyamada, M. Takeda, T. Sekine, T. Igarashi, H. Matsuo, Y. Kikuchi, T. Oda, K. Ichida, T. Hosoya, K. Shimokata, T. Niwa, Y. Kanai, and H. Endou. 2002. Molecular identification of a renal urate anion exchanger that regulates blood urate levels. Nature 417:447-452.

Fang, J., and M. H. Alderman. 2000. Serum uric acid and cardiovascular mortality: The NHANES I epidemiologic follow-up study, 1971-1992. JAMA 283:2404-2410.

Feig, D. I., D. H. Kang, and R. J. Johnson. 2008. Uric acid and cardiovascular risk. N. Engl. J. Med. 359:1811-1821.

Foell, D., H. Wittkowski, and J. Roth. 2007. Mechanisms of disease: A 'DAMP' view of inflammatory arthritis. Nat. Clin. Pract. Rheumatol. 3:382-390. 
Hayden, M. R., and S. C. Tyagi. 2004. Uric acid: A new look at an old risk marker for cardiovascular disease, metabolic syndrome, and type 2 diabetes mellitus: The urate redox shuttle. Nutr. Metab. (Lond.) 1:10.

Ioachimescu, A. G., D. M. Brennan, B. M. Hoar, S. L. Hazen, and B. J. Hoogwerf. 2008. Serum uric acid is an independent predictor of all-cause mortality in patients at high risk of cardiovascular disease: A preventive cardiology information system (PreCIS) database cohort study. Arthritis Rheum. 58:623-630.

Jackson, K. A., S. M. Majka, H. Wang, J. Pocius, C. J. Hartley, M. W. Majesky, M. L. Entman, L. H. Michael, K. K. Hirschi, and M. A. Goodell. 2001. Regeneration of ischemic cardiac muscle and vascular endothelium by adult stem cells. J. Clin. Invest. 107:1395-1402.

Kocic, G., L. Marinkovic, S. Stojanovic, G. Nikolic, and D. Stojanovic. 2011. Adsorption filter device for production of depurinised milk. G. Kocic, assignee. Serbia patent no. RS20100157 (A) 2011-04-30.

Kocic, G., G. Nikolic, D. Stojanovic, R. Pavlovic, T. Jevtovic-Stoimenov, D. Sokolovic, S. Stojanovic, S. Pajovic, and R. Kocic. 2010Technological procedure for production of composite dietetic milk beverage draught with reduced level of uric acid and purine. G. Kocic, assignee. Serbia patent no. RS20090231 (A) 2010-12-31.

Kocic, G., R. Pavlovic, G. Nikolic, D. Stojanovic, T. Jevtovic, D. Sokolovic, A. Cencic, S. Stojanovic, M. Jelic, and S. Zivanovic. 2012. The effect of depurinized milk draught diet on rat serum uric acid, lipid status and haematological parameters. J. Anim. Physiol. Anim. Nutr. (Berl.) 96:640-647.

Kocic, G., R. Pavlovic, G. Nikolic, A. Veljkovic, S. Panseri, L. M. Chiesa, T. Andjelkovic, T. Jevtovic-Stoimenov, D. Sokolovic, T.
Cvetkovic, S. Stojanovic, H. Kocic, and R. Nikolic. 2014. Effect of commercial or depurinized milk on rat liver growth-regulatory kinases, nuclear factor-kappa B, and endonuclease in experimental hyperuricemia: Comparison with allopurinol therapy. J. Dairy Sci. 97:4029-4042.

Marsche, G., S. Frank, A. Hrzenjak, M. Holzer, S. Dirnberger, C. Wadsack, H. Scharnagl, T. Stojakovic, A. Heinemann, and K. Oettl. 2009. Plasma-advanced oxidation protein products are potent high-density lipoprotein receptor antagonists in vivo. Circ. Res. 104:750-757.

Orlic, D., J. M. Hill, and A. E. Arial. 2002. Stem cells for myocardial regeneration. Circ. Res. 91:1092-1102.

Patschan, D., S. Patschan, G. Gobe, S. Chintal, and M. S. Goligorsky. 2007. Uric acid heralds ischemic tissue injury to mobilize endothelial progenitor . J. Am. Soc. Nephrol. 18:1516-1524.

Strasak, A. M., C. C. Kelleher, L. J. Brant, K. Rapp, E. Ruttmann, H. Concin, G. Diem, K. P. Pfeiffer, and H. Ulmer. 2008. Serum uric acid is an independent predictor for all major forms of cardiovascular death in 28,613 elderly women: A prospective 21-year follow-up study. Int. J. Cardiol. 125:232-239.

Tavil, Y., M. G. Kaya, S. O. Oktar, N. Sen, K. Okyay, H. U. Yazici, and A. Cengel. 2008. Uric acid level and its association with carotid intima-media thickness in patients with hypertension. Atherosclerosis 197:159-163.

Yagi, K. 1998. Simple procedure for specific assay of lipid hydroperoxides in serum or plasma. Methods Mol. Biol. 108:107-110. 